\title{
Trans-sulcal or fissure approach for supratentorial brain lesions: evaluation
}

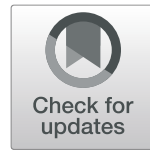

\author{
Ashraf Mohamed Farid ${ }^{*}$ (D), Ahmed Rizk Elkholy and Ebrahim Ahmed Shamhoot
}

\begin{abstract}
Object: To evaluate the efficacy and safety of trans-sulcal or fissure approach in surgical treatment of supratentorial lesions

Study design: Retrospective clinical case series

Methods: This study included 42 patients. Age ranged from 4 to 78 years. Sulcal dissection was done in 26 patients at or near the eloquent areas, and in 16 patients, it was done in non-eloquent areas. Diffusion tensor tractography, neuronavigation, and intraoperative monitoring were applied for lesions at the motor areas. The follow-up period was 24 months.

Results: Gross total excision could be achieved in 52\% of patients. Sulcal dissection was easy in 26 patients with the lax brain. The outcome, according to the Karnofsky scale, was 100 in 21/42 (50\%). Complications were transient deterioration of function in three patients, and immediate post-operative hemorrhage in two patients. Three patients became seizure-free after surgery.

Conclusion: The trans-sulcal dissection is a safe, useful, and applicable approach. Through which it is possible to reach deeply seated lesions. It preserves the motor functions, provides wider exposure, minimizes the need of brain retraction during surgery, and preserves the gyral layers. Complications are usually transient, specially in the presence of preoperative diffusion tensor tractography, intraoperative neuronavigation, and monitoring.
\end{abstract}

Keywords: Trans-sulcal, Supratentorial

\section{Introduction}

Anatomical studies considered the use of fissures and sulci as fundamental landmarks on the brain surface. Sulci could be used as corridors and have approximate depths ranging from 1 to $3 \mathrm{~cm}$. A sulcus can consist of one or more parts, which in some cases can be oriented in different directions [1].

Sulci and gyri are easily identified, particularly in standard magnetic resonance images (MRI), but their accurate intraoperative recognition is difficult because of their common anatomic variations and their arachnoid cerebrospinal fluid and vessel coverings. For practical purposes, the sulcal key points are located on the sulcus or the fissure where there is widening or dilatation. Those are important, specially their cranial-cerebral relationships [2].

\footnotetext{
* Correspondence: Aschraffarid@gmail.com

Neurosurgery Department, Tanta University, Tanta, Egypt
}

The transcortical trajectories usually traverse multiple layers of white matter tracts and associated with complications such as seizures, hemiparesis, memory loss, and visual field defects. Thus, it was concluded that the best approach is the shortest distance to the lesion with a perpendicular field of view that requires minimal retraction of the brain and avoids trajectory through important structures [3, 4].

The anatomical study of fiber dissection revealed that dissection of the invaginated cortex (the depth of the sulcus) uncovers only the arcuate fibers, which are short association fibers of the hemispheres located immediately beneath the cerebral cortex [5].

\section{Methodology \\ Patients data \\ This is a retrospective study conducted on 42 patients, 24 females, and 18 males. The age ranged from 4 to 78 years with a mean age of $47 \pm 3$ years. All patients were}


surgically treated by trans-sulcal approach for excision of supratentorial brain lesions. Patients were admitted and operated upon in neurosurgery department, Tanta University Hospital, in the period from May 2015 to August 2017. An approval from the research ethics committee of the Faculty of Medicine Tanta University [Reference Number: 32780] was obtained on 26/12/ 2018. Furthermore, being a retrospective study, patients' consents for participation and for publication were not applicable. Additionally, the co-authors had neither financial nor non-financial competing interest to report.

\section{Pre-operative planning}

Preoperative localization of the site of the lesion was through MRI [6]. We categorized the site of the lesion into eloquent, near eloquent, and non-eloquent based on preoperative MRI. Lesions at eloquent regions included the motor/sensory cortex, visual, temporal, and speech center. Near-eloquent regions were considered as immediately adjacent to eloquent structures. Non-eloquent regions included frontal, temporal polar, and right-sided parieto-occipital [7]. In patients with lesions at the motor areas, a super-added diffusion tensor tractography (DTT) was requested for completing the study. We expected tight brain pre-operatively, by the presence of perilesional edema in the CT or MRI, in addition to the mass effect; this resulted in the absence of the sulcal space. On the other hand, we expected a lax brain preoperatively by the absence of mass effect or edema and the presence of a sulcal space over the lesion, through which we might reach our target.

\section{Pre-operative preparations}

For lesions associated with perifocal edema, steroids were administered in a loading dose of $10 \mathrm{mg}$ intravenous (IV) and then $6 \mathrm{mg}$ IV every $6 \mathrm{~h}$, together with the anti-epileptics [8]. In patients with suspected metastatic lesions, workup was performed. An informed written consent was obtained prior to surgery.

\section{Procedures}

Mannitol (0.5-1 g/ $/ \mathrm{kg}$ body weight intravenously) during induction [9]. Intravenous anesthetics were preferable aiming at getting a lax brain. Sulcal dissection was dependent upon the intracranial pressure, as expected from MRI findings. In two of our patients who were suffering from a large intraventricular mass, we resorted to insert a ventriculoperitoneal shunt prior to definitive surgery. Patients with lesions at or near the eloquent areas, specially the motor cortex, we, first, localized the lesion using the navigator system (Brain $\mathrm{LAB}^{\circ}$, Munich, Germany) and then intraoperative confirmation. Intraoperative cortical stimulation and somatosensory evoked potential recordings were obtained and was carried out on patients with their lesions at the motor cortex (Inomed ${ }^{\bullet}$ Medizintechnik $\mathrm{GmbH}$, München, Germany).

Under high magnification, we started to dissect the arachnoid covering the sulcal vessels and we chose the wider corridor in relation to the vessel running between the two banks of the sulcus. In non-eloquent regions, occasionally a small vessel branching aside from the cortical vessel that was traversing the adjacent gyral banks could be cauterized without risk, this was better than being avulsed as a result of retraction. Care was taken not to coagulate any vessel supplying the white matter at the depth of the sulcus. Regarding lesions at the motor strip, we dissected the central sulcus through a distance more than the expected area of work (nearly $3 \mathrm{~cm}$ ) as this minimized the need of retraction if needed. We found it better to be on the post-central gyrus (on the sensory area, not the motor). We expected the extent of resection using the intraoperative navigation guidance.

Intraoperatively, we considered the herniation of the brain tissue beyond the dural edges as the tight brain, in which both adjacent gyral banks were effaced and the sulcal dissection was so difficult without the adequate measures for decreasing ICP. On the other hand, we defined the brain as being lax by the presence of a space between the cortex and the dural edges. Also, the gyral banks are not touching each other. So, sulcal dissection was easier.

\section{Post-operative care}

Intensive care unit (ICU) was ready for at least $24 \mathrm{~h}$. Routine post-operative computed tomography (CT) was done for all patients within $48 \mathrm{~h}$.

\section{Follow-up period}

The routine regular follow-up was carried out on both clinical and radiological basis (24 months).

\section{Statistical analysis}

The analysis was done using student $t$ test with IBM SPSS statistics for windows, Version 21 (IBM Corp, Armonk, NY).

\section{Results}

This study was conducted on 42 patients with different supratentorial brain lesions. All lesions were surgically excised using trans-sulcal or fissure approach.

Sulcal dissection was done at or near eloquent areas in 26 patients $(61.9 \%)$ including central sulcus dissection in five patients (Fig. 1 and 2), dissection near the motor cortex in six patients, at the temporal lobe in ten patients, at the left posterior perisylvian area to reach a lateral ventricular neoplasm in one patient, inferior frontal sulcus dissection (near the speech area) in one patient, and Sylvian fissure dissection in three patients. 


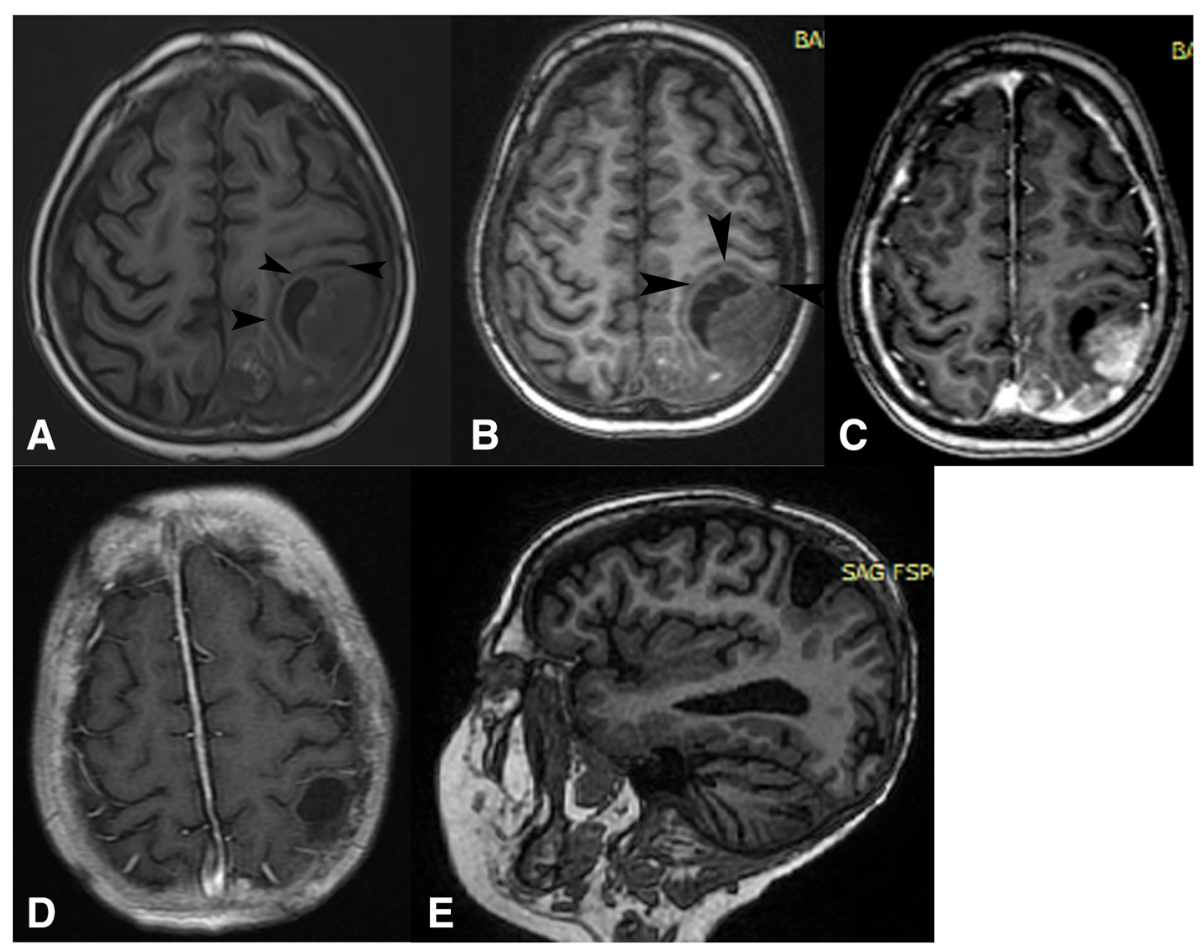

Fig. $\mathbf{1} \mathbf{a}$ and $\mathbf{b}$ axial non-contrast T1WI. The arrowheads point to the sulcal approach. $\mathbf{c}$ represents axial post-contrast MRI. $\mathbf{d}$ and $\mathbf{e}$ are 18-month post-operative axial and sagittal MRI showing healthy gyral banks

Sulcal dissection was done at non-eloquent areas in 16 patients (38.1\%) which included frontal, temporopolar, and parieto-occipital regions.

The length of sulcal dissection among patients of this study was nearly $3 \mathrm{~cm}$, and the gyral width was nearly 2 $\mathrm{cm}$ which permitted surgical manipulation with minimal brain retraction in all patients. The sulcus planned to be dissected was continuous in 18 patients (42.9\%) and interrupted in 24 patients (57.1\%). There was no statistically significant difference between both types in the extent of resection ( $P=0.314$ and 0.236 , respectively) (Table 1).

Sulcal dissection was easy in $26(61.9 \%)$ with the lax brain, and it was difficult in 16 patients (38.1\%) with the tight brain. There was a statistically significant difference in the extent of resection between the tight and lax brain as gross total resection was higher in the lax brain $(P=0.01)$ (Table 2).

Diffusion tensor tractography was done preoperatively in patients with lesions at the motor cortex (five patients), and the pyramidal tracts were displaced by the lesion in all of these patients. Sulcal dissection was done at the motor cortex in five patients and near the motor cortex in six patients (total of 11 patients); gross total resection could be achieved in six patients $(6 / 11,54.5 \%)$ and subtotal resection could be achieved in five patients $(5 / 11,45.5 \%)$. Deterioration in function was found after surgery in two patients $(2 / 5,40 \%)$ in the form of

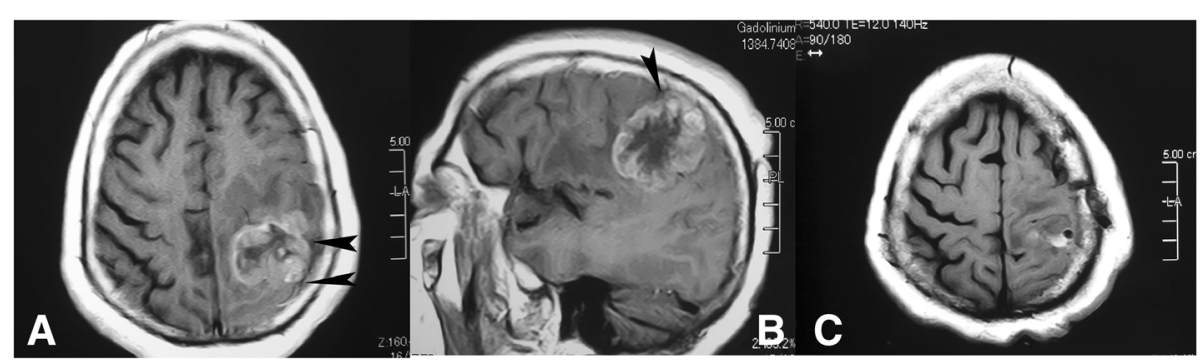

Fig. 2 a Axial and $\mathbf{b}$ sagittal post-contrast MRI showing a neoplasm at the motor area. Arrowheads point to the sulcal approach. $\mathbf{c}$ Early postoperative axial MRI 
Table 1 Relation between the extent of resection and the sulcal pattern

\begin{tabular}{lll}
\hline Sulcus type & Gross total & Subtotal \\
\hline Continuous & $10(55.6 \%)$ & $8(44.4 \%)$ \\
Interrupted & $12(50 \%)$ & $12(50 \%)$ \\
$P$ & 0.314 & 0.236 \\
\hline
\end{tabular}

transient deterioration in the motor power from grade $\mathrm{V}$ to grade IV which resolved completely in 3 months. There was a statistically significant difference between gross total and subtotal resection at and near the motor cortex in relation to the functional outcome as the deterioration of function was found only among patients with gross total removal $(P=0.01)$ (Table 3$)$.

Trans-sulcal dissection was used to excise intraventricular lesions in two patients; one of them is located in the atrium of the lateral ventricle and sulcal dissection was done at the left temporoparietal region. This patient developed post-operative transient dysphasia. The second patient had a lateral ventricular mass near the frontal horn, and sulcal dissection was done at left inferior frontal sulcus near speech area but without any post-operative speech dysfunction.

Gross total excision could be achieved in 22 patients $(22 / 42,52 \%)$ while in 20 patients $(20 / 42,48 \%)$, subtotal excision could be achieved. The outcome was measured according to the Karnofsky scale. It was 100 in 21/42 (50\%), 90 in $8 / 42(19 \%), 80$ in $4 / 42(10 \%)$, and less than 80 in $9 / 42(21 \%)$.

Complications were immediate post-operative hemorrhage in two patients necessitated urgent evacuation. Histopathology revealed 34 patients with glial neoplasms, two cavernomas, five metastatic deposits, and one cortical dysplasia. Patients with glial neoplasms were 28 glioblastoma multiforme, three astrocytomas, one ganglioglioma, one dysembryoblastic neuroectodermal tumors (DNET), and lastly, one patient with a central neurocytoma grade II (Table 4).

It is worthy to mention that patients who had cavernous malformation and the patient who had cortical dysplasia completely cured from seizures (Table 4), in whom they were the main presentation and no longer use of antiepileptic medication up to 1.5 year follow-up, while the patient who had ganglioglioma was controlled on monotherapy rather than polytherapy.

Table 2 Relation between degree of brain relaxation and the extent of resection

\begin{tabular}{lll}
\hline Extent of resection & Tight & Lax \\
\hline Gross total & $3(13.6 \%)$ & $19(86.4 \%)$ \\
Subtotal & $13(65 \%)$ & $7(35 \%)$ \\
$P$ & $0.021^{*}$ & $0.01^{*}$ \\
\hline
\end{tabular}

Table 3 Relation between sulcal dissection at and near the motor cortex and functional preservation

\begin{tabular}{lll}
\hline Extent of resection & Preservation of function & Deterioration of functions \\
\hline Gross total & $4(66.7 \%)$ & $2(33.3 \%)$ \\
Subtotal & $5(100 \%)$ & $0(0.0 \%)$ \\
$P$ & 0.234 & $0.01^{*}$ \\
\hline
\end{tabular}

\section{Discussion}

The distance from the crown of the gyrus to a subcortical lesion is double the distance measured from a sulcal depth [10]. Based upon this idea, we have planned this study.

Different studies documented the usefulness of integrating the preoperative diffusion tensor tractography into the neuronavigation system to localize the descending pathway of eloquent areas during the surgical procedure [3], and so, the better functional outcome could be achieved.

In this work, we used preoperative diffusion tensor tractography for patients with lesions at the motor cortex (five patients) in addition to the use of intraoperative neuronavigation. Transient deterioration in motor function was found in two patients $(2 / 5,40 \%)$ which resolved completely in 3 months. So, we reported that it was useful not only in localizing the eloquent areas but also to predict whether the pyramidal tracts were displaced or infiltrated by the lesions. This is considered as an important prognostic factor and essential for explanation to the patient [11]. Salcman [12] reported that the maximum safe gyral incision at the eloquent brain region must be not more than $3 \mathrm{~cm}$ and vertical to the gyrus to prevent deterioration in the functional state.

In this study, we used the trans-sulcal approach in all patients. The length of sulcal dissection was about $3 \mathrm{~cm}$, and the gyral width was nearly $2 \mathrm{~cm}$. We did not use self-retaining retractors in any patient, so, we recommended the trans-sulcal dissection as it can provide enough working length and good exposure for surgical manipulation with minimal brain retraction.

In our series, sulcal dissection was easy in the lax brain and gross total resection of the lesion was higher than that in the tight brain. So, we agree with the results of Piepmeier et al. [13] who reported that swollen brain and effaced sulci are possible obstacles during surgery and usually associated with difficulty in gross total resection.

Different studies evaluated the use of trans-sulcal dissection in surgical treatment of lesions at and near

Table 4 Relation to the extent of resection and the histopathological pattern

\begin{tabular}{lllll}
\hline Extent of resection & Glial neoplasms & Mets & Cavernomas & Dysplasia \\
\hline Gross total & $16(72.7 \%)$ & $3(13.6 \%)$ & $2(9.1 \%)$ & $1(4.5 \%)$ \\
Subtotal & $18(90 \%)$ & $2(10 \%)$ & $0(0.0 \%)$ & $0(0.0 \%)$ \\
\hline
\end{tabular}


the motor cortex. They reported that it was an applicable approach as it usually associated with little complications and good functional outcome [14-16]. It was the same in our study as sulcal dissection was done in 11 patients at and near the motor cortex, and there were two patients with post-operative transient deterioration in the motor power. This was mainly related to gross total resection in these patients.

Ramakrishna et al. [17] reported that in $27 \%$ of their patients, $50-90 \%$ of the size of glial neoplasms could be resected even with the use of functional MRI and intraoperative mapping. In our study, we used preoperative DTT, intraoperative neuronavigation, and intraoperative monitoring, gross total excision could be achieved in 22 patients (52\%) and subtotal excision could be achieved in 20 patients $(48 \%)$. We attributed these results to different factors which affected the extent of resection including the nature of the lesion, size, vascularity, working in a lax or tight brain, and the location of the lesion (eloquent or non-eloquent).

Piepmeier et al. [13] and Abosch et al. [18] described various complications resulted from gyral incision to reach ventricular mass such as motor deficit, visual deterioration, speech dysfunction, seizures, and incomplete resection. They reported that working through the shortest pathway which does not affect the neurological function is preferred during surgery of intraventricular tumors. So, they recommended the use of the trans-sulcal approach.

Wen et al. [15] stated that the inferior frontal sulcus is a suitable corridor to reach deeply seated neoplasms; moreover, they could reach the frontal horn through this way. Kawashima et al. [19] and Bertalanfly and Mahmoodi [20] advocated the use of superior frontal sulcus, interparietal sulcus, and occipitotemporal sulcus for various ventricular masses. They reported that the drawbacks of the transcortical approach are injury to both long, short association fibers and pyramidal fibers. Also there is a limited cortical incision to avoid more injury, which may limit the exposure.
In our study, we used the trans-sulcal approach (the shortest way) to expose and excise intraventricular masses in two patients; in one of them, the sulcal dissection was done through the left temporoparietal region to excise intraventricular lesion located in the atrium of the lateral ventricle and this patient developed transient post-operative dysphasia. In the other patient, the sulcal dissection was done through the left inferior frontal sulcus to excise intraventricular lesion located near the frontal horn without any post-operative speech dysfunction.

Our results of surgery of cavernous lesions operated through trans-sulcal technique matched with those of Wanebo and Kris [21]. Our patients cured from seizures and became completely independent of antiepileptic medications (Fig. 3).

There are different factors that should be taken in consideration to avoid complications during trans-sulcal approach and to improve the outcome such as the use of preoperative DTT in addition to intraoperative neuronavigation and functional mapping and avoid working on the tight brain and subtotal resection may be of value in patients with lesions at eloquent brain areas.

The limitations of this study are being retrospective and limited number of patients.

\section{Conclusion}

The trans-sulcal dissection is a safe, useful, and applicable approach in brain surgery. Deeply seated lesions could be reached by this approach through a short way. It preserves the motor functions, provides wide exposure, minimizes the need of brain retraction during surgery, preserves the gyral layers, and usually associated with good functional outcome and little complications specially in the presence of preoperative DTT, intraoperative neuronavigation, and brain mapping.

\section{Abbreviations}

CT: Computed tomography; DNET: Dysembryoblastic neuroectodermal tumors; DTT: Diffusion tensor tractography; ICU: Intensive care unit; MRI: Magnetic resonance imaging

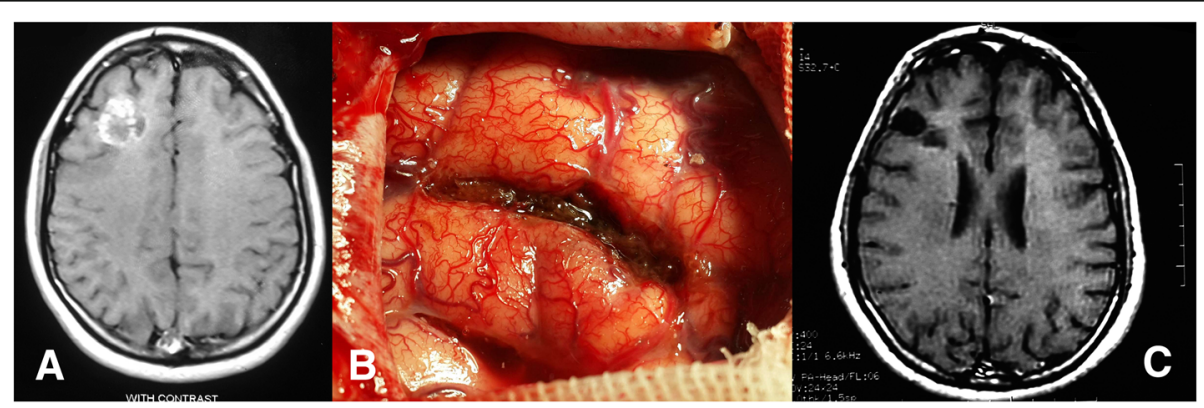

Fig. 3 a Axial T1 MRI, right frontal Cavernoma. b The operated site showing the sulcal dissection after lesion removal and the sulcal cavity is filled with a hemostatic agent. c Axial T1 MRI eight-month post-operatively 


\section{Acknowledgements}

The authors sincerely thank El-Mahalawy $\mathrm{M}$ and all of the department members of Neurosurgery, Tanta University Hospital, for their help in doing this work.

\section{Authors' contributions}

All authors equally participate in operating patients, data collection, data analysis, and scientific writing. All authors read and approved the final manuscript.

\section{Funding}

There was no funding for this study.

\section{Availability of data and materials}

The datasets used and/or analyzed during the current study are available from the corresponding author on reasonable request.

\section{Ethics approval and consent to participate}

Approved from the research ethics committee of the Faculty of Medicine at Tanta University [reference number 32780 obtained on 26/12/2018]. Consent for participation was not applicable as this is a retrospective study, so, the ethics committee waived the consent to participate.

\section{Consent for publication}

Not applicable.

\section{Competing interests}

The authors declare that they have no competing interests.

Received: 24 January 2019 Accepted: 1 August 2019

Published online: 26 August 2019

\section{References}

1. Ribas GC. The cerebral sulci and gyri. Neurosurgical Focus. 2010;28(2):E2.

2. Alexandre $Y$, Eduardo C, Koshiro N, Aldo JR. Surgical anatomy of microneurosurgical sulcal key points, Guilherme C. Ribas, M.D. Neurosurgery. 2006; 59[ONS Suppl 4]: ONS-177-ONS-211.

3. Fernández M, Rhoton A, Juan ÁL, Yukinari K, Chanyoung C, de Oliveira EP. Three-dimensional microsurgical and tractographic anatomy of the white matter of the human brain. Neurosurgery. 2008; 62 [SHC Suppl 3]: SHC-989SHC-1027.

4. Greenberg MS: Primary tumors of the nervous and related systems: tumors of the neuroepithelial tissue, in Handbook of Neurosurgery: Greenberg MS (ed), Thieme Medical Publisher, New York, NY, USA, 2016; 758.

5. Türe U, Yaşargil MG, Friedman AH, Al-Mefty O. Fiber dissection technique: lateral aspect of the brain. Neurosurgery. 2000;47:417-27.

6. Hinojosa AQ, Ojemann SG, Sanai N, Dillon WP, Berger MS. Preoperative correlation of intraoperative cortical mapping with magnetic resonance imaging landmarks to predict localization of the Broca area. J Neurosurg. 2003:99:311-8.

7. Grabowski M, Recinos BS, Pablo F, Nowacki AS, Schroeder JL, Angelov L, Barnett GH, Vogelbaum MA. Residual tumor volume versus extent of resection: predictors of survival after surgery for glioblastoma, Clinical article. J Neurosurg. 2014; 121: 1115-1123.

8. Greenberg MS. Steroids in brain tumors. In: Handbook of Neurosurgery, Greenberg MS (ed), Thieme Medical Publisher, New York, USA, 2010; pp. 588.

9. Salcman M: The surgical management of Gliomas. In: George T. Tindall, Paul Cooper and Daniel L. Barrow (eds), The Practice of Neurosurgery, 1997; Vol. 1, Chapt 45, pp. 649-70.

10. Campero A, Tróccoli G, Martins C, Fernandez JC, Yasuda A, Rhoton A. Microsurgical approaches to the medial temporal region: an anatomical study. Neurosurgery. 2006; 59[ONS Suppl 4]: ONS-279-ONS-308.

11. Carpenter MB. Core text of neuroanatomy, 4th eds., Lippincott-Williams and Wilkins, 1991; Chapt 9, pp. 286.

12. Salcman M. High grade cerebral hemisphere gliomas (adults). In: Kaye A and Black P (eds), Operative Neurosurgery, 2000; Vol. 1, Chapt 26, pp. 317-332.

13. Piepmeier JM, Spencer DD, Sass KJ, George TM. Lateral ventricular masses. In: Apuzzo MLJ (eds), Brain Surgery; Complication Avoidance and Management, 1993; Vol. 2, Chapt 19, pp. 581-600.
14. Robles GS, Duffau H. Surgical management of World Health Organization Grade II gliomas in eloquent areas: the necessity of preserving a margin around functional structures. Neurosurg Focus. 2010;28(2):E8.

15. Wen HT, Rhoton ALJr, Mussi ACM. Surgical Anatomy of the Brain. In: "Youmans JR and Winn (ed.), Neurological Surgery", 7th ed. Philadelphia: Elsevier Inc.; 2017. Vol. 1, pp. 49-65.

16. Patel AJ, Lang FF, Suki D, Wildrick DM, Sawaya R. Metastatic Brain Tumors. In: "Youmans JR and Winn (ed.), Neurological Surgery", 7th eds. Philadelphia: Elsevier Inc.; 2017. Vol. 1, Chapt 146, pp. 1097.

17. Ramakrishna R, Hebb A, Barber J, Rostomily R, Silbergeld D. Outcomes in reoperated low-grade gliomas. Neurosurgery. 2015;77:175-84

18. Abosch A, McDermott MW, Wilson ChB. Lateral ventricular tumors. In: Kaye A and Black P (eds.), Operative Neurosurgery, 2000; Vol. 2, Chapt 64, pp. 799-812.

19. Kawashima M, Li X, Rhoton A, Ulm AJ, Oka H, Fujii K. Surgical approaches to the atrium of the lateral ventricle: microsurgical anatomy. Surg Neurol. 2006; 65:436-45.

20. Bertalanffy $\mathrm{H}$ and Mahmoodi R. Ventricular tumors. In: Youmans JR and Winn (eds.), Neurological Surgery, $7^{\text {th }}$ ed., Elsevier Inc., 2017; Vol. 1, Chapt 153, pp 2394-2395.

21. Wanebo J, Lanzino G, Zabramski JM, Spetzler RF. Supratentorial cavernous malformations, operative techniques. Neurosurgery. 2002:5(3):176-84.

\section{Publisher's Note}

Springer Nature remains neutral with regard to jurisdictional claims in published maps and institutional affiliations.

\section{Submit your manuscript to a SpringerOpen ${ }^{\circ}$ journal and benefit from:}

- Convenient online submission

- Rigorous peer review

- Open access: articles freely available online

- High visibility within the field

- Retaining the copyright to your article

Submit your next manuscript at $\boldsymbol{\nabla}$ springeropen.com 